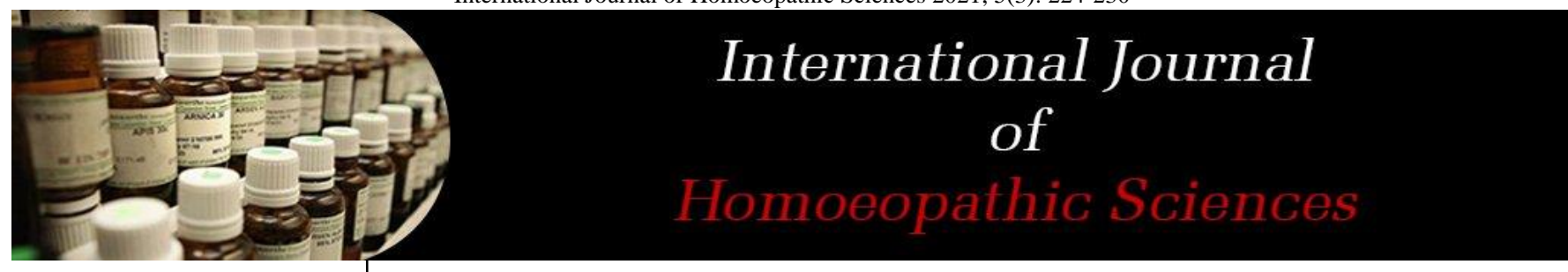

E-ISSN: 2616-4493 P-ISSN: 2616-4485 www.homoeopathicjournal.com IJHS 2021; 5(3): 224-230 Received: 09-05-2021 Accepted: 12-06-2021

\section{Dr. Deepthi Gilla}

Research Officer $(\mathrm{H}) /$ Assistant

Professor, Department of

Psychiatry, NHRIMH,

Kottayam, Kerala, India

\section{Dr. Dhanraj Kumar Rana} Research Officer (H)/Assistant Professor, Department of Psychiatry, NHRIMH, Kottayam, Kerala, India

\section{Dr. Deepak KP}

Post Graduate Scholar,

Department of Psychiatry,

NHRIMH, Kottayam, Kerala, India
Corresponding Author: Dr. Deepthi Gilla Research Officer (H)/Assistant Professor, Department of Psychiatry, NHRIMH, Kottayam, Kerala, India

\title{
Application of mental status examination in homoeopathic prescribing
}

\section{Dr. Deepthi Gilla, Dr. Dhanraj Kumar Rana and Dr. Deepak KP}

\author{
DOI: https://doi.org/10.33545/26164485.2021.v5.i3d.427
}

\begin{abstract}
Homoeopathy is a developing system of medicine which is based on the principle of individualization. Understanding the personality traits and mental symptoms of an individual is pivotal in homoeopathic prescribing. Eliciting mental symptoms requires skillful application of art and science. Mental status examination (MSE) is a structured assessment of the patient's behavioural and cognitive functioning, usually administered in psychiatry but in brief is a part of every medical exam. The components of MSE can be utilized in homoeopathy to elicit certain mental symptoms, erect appropriate rubrics and to arrive at the right similimum, which is indispensable for cure.
\end{abstract}

Keywords: homoeopathy, mental status examination (MSE), repertory, organon of medicine

\section{Introduction}

Homoeopathy is a developing, holistic system of medicine which is based on the principle of individualization and prescriptions based on totality of symptoms. The importance of mental symptoms in homoeopathic prescribing cannot be over-emphasized. Understanding the personality of an individual is pivotal for this reason. Hence eliciting mental symptoms requires skilful application of art and science in different methods and techniques.

The Mental Status Examination (MSE) is a structured assessment of the patient's behavioral and cognitive functioning. It includes descriptions of the patient's appearance and general behavior, level of consciousness and attentiveness, motor and speech activity, mood and affect, thought and perception, attitude, judgment and insight, the reaction evoked in the examiner, and, finally, higher cognitive abilities. The specific cognitive functions of alertness, language, memory, constructional ability, and abstract reasoning are the most clinically relevant ${ }^{[1]}$.

The Mental Status Examination (MSE) is a core element of the psychiatric assessment ${ }^{[2]}$. It is compared with physical examination as a reliable method of objective data investigation [3]. The history and MSE are the most important diagnostic tools, a psychiatrist have to obtain information to make an accurate diagnosis. Although these important tools have been standardized in their own right, they remain primarily subjective measures that begin the moment the patient enters the office. The mental status examination includes general observations made during the clinical encounter, as well as specific testing based on the needs of the patient and physician ${ }^{[4]}$.

The MSE is an important component of all medical exams and may be viewed as the psychological equivalent of the physical exam. Mental State Examination (MSE) is compared with physical examination as a reliable method of objective data investigation [5]. It provides a format for the systematic observation and recording of information about a person's thinking, emotions, and behavior. These data combined with information from the history are the basis for formulating a differential diagnosis. As is true for the physical examination, a physician conducting a mental status examination notes only those findings present at the time of interview. Historical information is excluded. A patient may report having had auditory hallucinations the day before, but unless they are present when the examination is conducted, hallucinations are not recorded in the mental status examination. The physician must also be as objective as possible in making mental status observations. To investigate the current level of psychopathological knowledge, the reliability of the MSE can be used as a proxy as it is based on descriptive psychopathology. The MSE also allows you to assess patients' risk of harm to themselves or others or both. When conducting an MSE, it is important to write down patients' words and the order in which they are being expressed 
verbatim, to avoid them being misinterpreted ${ }^{[6]}$. Familiarity with the components of the examination can help physicians evaluate for and differentiate psychiatric disorders ${ }^{[7]}$.

Since 1980 the various editions of DSM have emphasized descriptive phenomenology in making psychiatric diagnoses to the exclusion of inferred, intra-psychological processes, in an explicit attempt to enhance diagnostic reliability. Observed data are always more reliable than inferred data. Correspondingly, the mental status examiner should strive to record findings that are as free of interpretation or inference as possible. The formal organization of the mental status examination ensures completeness. In the actual interview of a psychiatric patient it is seldom necessary to proceed with an inflexible, prescribed series of questions. Much of the mental status examination is observational and can be made in the course taking the history. There are several specific tests of cognitive function, but much of this information can be obtained simply by talking with a patient. The experienced clinician does several things simultaneously in conducting a psychiatric interview: establishing rapport, eliciting important historical information, recognizing areas of greater or lesser emotional intensity, and making ongoing mental status observations ${ }^{[8,}$ 9].

Kent's hierarchy of mental symptoms as Will/ Emotions, Understanding/ Intellect and Memory helps to some extent to arrive at the mental generals comprehensively ${ }^{[10]}$. This is an attempt to explore homoeopathic perspective of MSE and correlate the various elements and components of MSE to Homoeopathic Rubrics for better understanding and incorporating into homoeopathic prescribing.

\section{Aphorisms Related to MSE}

In $\S 213$, Organon of Medicine, Master Hahnemann clears emphasizes the importance of mental generals in everyone. We shall, therefore, never be able to cure conformably to nature - that is to say, homoeopathically - if we do not, in every case of disease, even in such as are acute, observe, along with the other symptoms, those relating to the changes in the state of the mind and disposition, and if we do not select, for the patient's relief, from among the medicines a disease-force which, in addition to the similarity of its other symptoms to those of the disease, is also capable of producing a similar state of the disposition and mind ${ }^{[11]}$.

In Footnote to Aphorism 213, He says "Thus aconite will seldom or never effect a rapid or permanent cure in a patient of a quiet, calm, equable disposition: and just as little will nux vomica be serviceable where the disposition is mild and phlegmatic, pulsatilla where it is happy, gay and obstinate, or ignatia where it is imperturbable and disposed neither to be frightened nor vexed."

Hahnemann has given a brief insight to MSE in $\S 90$, Organon of medicine, $5^{\text {th }}$ edition

When the physician has finished writing down these particulars, he then makes a note of what he himself observes in the patient 1 , and ascertains how much of that was peculiar to the patient in his healthy state. For example, how the patient behaved during the visit - whether he was morose, quarrelsome, hasty, lachrymose, anxious, despairing or sad, or hopeful, calm etc. Whether he was in a drowsy state or in any way dull of comprehension: whether he spoke hoarsely, or in a low tone, or incoherently, or how otherwise did he talk? What was the color of his face and eyes, and of his skin generally? What degree of liveliness and power was there in his expression and eyes? whether he lay with head thrown back, with mouth half or wholly open, with the arms placed above the head, on his back, or in what other position? what effort did he make to raise himself? and anything else in him that may strike the physician as being remarkable.

$\S 153$, Organon of medicine, Master directs that the more striking, singular, uncommon and peculiar (characteristic) signs and symptoms ${ }^{[1]}$ of the case of disease are chiefly and most solely to be kept in view in search for a homoeopathic specific remedy, in contrast to the more general and common ones. Hence while considering a definite rubric under each component depends on whether it is Common or Uncommon in that given case. For example- Smiling- may be common in Schizophrenia, BPAD, Dementia, etc. MR, whereas in other disorders like OCD, GAD, Phobias, Autism etc., it is uncommon. One has to observe which part of MSE is not correlating with the rest of symptoms which lead to diagnosis. Although most features of MSE may appear to be common symptoms in psychiatric conditions, they may be considering in prescribing when they are Presenting, Prominent and Persistent. Apart from being useful in making a psychiatric diagnosis, MSE is also useful for eliciting mental symptoms.

\section{Components of MSE}

Some of the components are objectively derived whereas few questions are subjective and some are derived in both ways. Different Components of MSE are listed in Table no. 1. Various components are explained below with examples of repertorial representation and corresponding rubrics.

1. General Appearance and Behaviour (GAB): This section of examination archives findings related to the patient's levels of cooperativeness and behavioral activation, manner of relatedness and interaction and state of grooming and dress. Ex: The patient appeared her stated age, was dressed eccentrically and was poorly groomed. She seemed tense, was distracted and difficult to structure, but was otherwise cooperative with the interview. The following things should be considered while writing GAB.

- Hygiene: clean, body odor, shaven, grooming

- Gait, posture, attitude

- Dress: clean, dirty, neat, ragged, climate appropriate anything unusual?

- Jewellery: rings, earrings - anything unusual?

- Makeup: lipstick, nail polish, eye makeup - anything unusual?

- Other: prominent scars, tattoos

\section{Rubrics ${ }^{[12]}$ related to $\mathbf{G A B}$ are}

- Mind, Dress, Aversion to (Con, sulph),

- Mind, Dress, Conservative(kali-c)

- Mind, Indecently dresses

- Mind, Indifference, appearance, to his personal- sulph

- Foppish (Concerned with his clothes and appearance in an affected and excessive way.)

- Fastidious

- Mind, Smiling

- Mind, Frown, disposed to-

- Mind- Eyes, downcast, walking with the eyes- Cham

- FACE, Expression: Astonished, Bewildered, Confused, Happy, Haggard, Idiotic, Serious, Vacant, Absent etc.

- MIND- Frown, disposed to 
- Extremities, Ataxia

- Extremities, Tottering

- Extremities, stand cannot,

- $\quad$ standing difficult

- $\quad$ standing impossible

- stepping high

- Generalities, SIT, sits, ground looking at -stram

- legs cross, uncross cannot- bell, ther

- $\quad$ sit, inability to sit- glon, lyco, naja, stram

- Attitude:

- Secretive, Confiding

- Suspicious

- Irritable

- Mocking, Sarcastic- CV, Graph

2. Psychomotor Activity (PMA): This section of the examination offers observations about the patient's general level of motor activity, posture and gait and identifies the presence of abnormal movements such as tics, mannerisms, posturing, choreic, athetoid, and dyskinetic movements. PMA is described in terms of

1. Appropriateness: Appropriate to situation or not

2. Quantity: Normal, Increased (eg: in mania, agitation, hyperkinetic syndrome, drug induced akathisia manifested as foot tapping, scalp rubbing, hand wringing, pacing, alternatively sitting and standing and picking at clothing or bed linens). Decreased (eg: in severe depression, parkinsonism, some schizophrenic patients expressed as excessive speech latency or stupor (mute, immobile, unresponsive to givorous painful stimuli)

3. Quality: facial movements eg: in oral dyskinesia, tremors in hands or body eg: in anxiety neurosis or neuroleptic induced

4. Mannerisms ie: odd, repetitive movements, may be a part of a goal directed activity (eg: in normal persons, manics)

5. Stereotypies ie: motor or verbal repetition without any discernible goal eg: in schizophrenics

6. Automatic obedience: a type of catatonic behavior when verbal instructions are overridden by tactile or visual stimuli eg: the patient shakes hands with the examiner, contrary to the firm verbal instructions whenever the examiner's right hand is extended eg: in Schizophrenia.

7. Mitmachen: despite instructions to be contrary, the patient will allow a body part to be put into any position without resistance to the light pressure, then returns the body part to the original position when the examiner releases it.

8. Echopraxia: automatic copying of the examiner's movements or postures. eg: In catatonic schizophrenia

9. Cholalia: automatic repetition of the examiner's utterances, eg: in catatonic schizophrenia, Autism.

10. Catalepsy: prolonged sustaining of an awkward posture or position. Eg: in schizophrenic patients.

\section{Rubrics Related To Pma}

- Mind- Activity desires

- Mind-Dancing

- Mind-Counting continually

- Mind-Grimaces

- Mind-Gestures, makes, automatic
3. Speech

- Intensity: audible, excessively loud (eg: in excited manic or schizophrenic), soft (eg: withdrawn depressives)

- Pitch: monotonous (eg: retarded patients), abnormal changes (eg: in manic patients)

- Speech: slow (eg: in depressives), rapid (eg: in manic patient), pressure (i.e: difficult to interrupt, eg: in mania)

- Ease of speech: under much pleasure, hesitatingly (eg: in manic patients)

- Spontaneity: spontaneous (eg: manic), only when asked (eg: obsessive patients), mute

- Productivity: usual verbal productivity, garrulous or laconic (eg: manic patients)

- Relevance: relevant, flighty (eg: manic patients), distractible (eg: mania)

- Manner: excessively formal ( eg: paranoid patients), relaxed, inappropriately familiar(eg: mania)

- Deviation: Neologism (coining of new words or used words inappropriate way)

- Echolalia: (automatic repetition of the examiner's utterances) eg in Scizophrenia, Autism

- Clang association: (speech in which sounds rather than meaningful conceptual relationships govern words choice) eg: mania

\section{Rubrics related to speech}

- Mind - Speech, Abrupt $(=$ sudden and unexpected $)$ Babbling ( $=$ the action or fact of talking rapidly and continuously in a foolish, excited and incomprehensible way)

- Bombast(=high sounding but with little meaning, inflated), Hasty, heavy, repeat, unintelligible, vivacious $=$ attractively and lively

- Mouth-Speech, swallowing his words- Cic, Staph, thuj

- Mouth-Speech, Thick, slurred

- Mouth-Speech, Repeats, everything said to him, ... the same thing

4. Mood and Affect: The terms mood and affect vary in their definition such that one term often merges with the other. A number of authors have recommended combining the two elements into a new label "emotional expression".

Traditionally Mood is defined as the patient's internal and sustained emotional state. Its experience is subjective and hence it is best to use the patient's own words when describing mood. Terms such as normal, calm, sad, happy, angry, irritable, and anxious are eg of descriptors patients may offer Affect differs from mood in that it is the outward manifestation of a mood state visible to others.

1. Appropriateness (quality): it refers to affective display to the content of speech and thought. Inappropriate affect is characteristic of Schizophrenia (where it is also known as incongruous affect). Affect may be inappropriate and labile in psedo-bulbar palsy in which the patients laugh while describing depression or cry while claiming to be happy. Affect may be shallow and inappropriate in frontal lobe lesions

2. Intensity (quantity): normal or increased as in mania or Decreased when the patients appear shallow and vacuous with little conviction in their statements eg: in Schizophrenia.

3. Type: elated (eg: in mania), sad (eg: in depression), 
fear (eg: in phobia), anger (eg: in S), anxious (eg: in anxiety neurosis), irritable (eg: in hypomania), blunting (affects are diminished in intensity and constricted to a narrow neutral range, eg: in Schizophrenia), flat (no effective response at all, eg: in Schizophrenia. Motor dysprosody following lesions in anterior right cerebral hemisphere strikes with monotonous sparse speech without affective coloring.

4. Range: constricted (when a spectrum of moods not elicitable, eg: in depression), expanded (excesses of joyfulness or sadness are seen, eg: in mania

5. Stability: stable (eg: in depression). Lability (eg: in un understandable, rapid changeability, eg: in organic mental disorders). Lability with inappropriateness, eg: in pseudobulbar palsy. Relatedness (ability to establish rapport and inter-personal connectedness

\section{Rubrics related to $\operatorname{mood}$ and affect}

- Mind, Mood- agreeable, alternating, changeable, contradictory, Holy (Olib sac), Insupportable- calc, lachrymose, Repulsive (PULS).

- Hopeless- Despair

- Ecstasy- Acon, Phos

- Elated

- Euphoria

- Depressed

- Sadness (749)

- Apathy- Indifference

- Anhedonic- Pleasure, Nothing in-Discontent, everything

- Haughty, Contemptuous- CIC, PLAT

5. Thought: Cognition refers to an individual's thoughts, knowledge, interpretation, understanding and ideas about himself and his environment. Thought (thinking) is the goaldirected arousal of symbols, ideas and associations leading to a reality-oriented conclusion. The thought comes to light through verbalization (talk/speech), writing and gestures. Normally, thinking is coherent (fully understandable) and relevant to the context.

\section{Abnormalities of thought}

\section{i). Thought Process/Form (Given by Cameron)}

1. Asyndesis: Lack of adequate connections between successive thoughts. The patient uses clusters of more or less related thoughts in place of well-knit sequences.

2. Metonyms: Imprecise expressions or use of substitute term or phrase instead of more exact one. (eg: for a penwriting stick)

3. Interpretation of themes: the patient's speech contains elements which belong to the task in hand interspersed with a stream of phantasy which he cannot understand.

4. Overinclusion: inability to maintain the boundaries of the problem and to restrict operations within their correct limits. The patient shifts from one hypothesis to another

The disorder of form of thinking in schizophrenia are

a. Derailment: the thought slides on to a subsidiary thought.

b. Substitution: a major thought is replaced by a subsidiary one.

c. Omission: senseless omission of a thought or part of it.

d. Fusion: heterogenous elements of thoughts are interwoven.

e. Driveling: disorganized intermixture of constituent parts of one complete thought.

f. Desultory thinking: speech is grammatically and syntactically correct but sudden ideas force their way in from time to time.

ii) Thought Flow/Stream

a) Disorders of Tempo

1. Flight of ideas: Rapid speech with quick changes of ideas that may be associated with chance factors such as by the sound of the words but which can usually be understood, eg: (see the king is standing, king, king, sing, sing, bird on the wing, wing, wing), eg: in mania

2. Prolixity: In hypomania there is ordered flight of ideas, i.e: despite many irrelevances, the patient is able to return to the task in hand. It is also called Prolixity

3. Inhibition/retardation: thoughts are slowed down and the number of ideas and mental images which present themselves are decreased, eg: in depression, stupor

4. Circumstantiality: thinking proceeding slowly with many unnecessary trivial details but finally the goal is reached, eg: in mania, organic mental disorders, schizophrenia.

5. Tangentiality: the final goal is not reached and the patient loses track of the original question.

6. Incoherence: marked degree of loosening of associations in which the patient shifts ideas from on to another with no logical connection, accompanied by a lack of awareness on the part of the patient that ideas are not connected. The examiner cannot understand the talk. It is seen in schizophrenia

\section{b) Disorders of Continuity}

- Perseveration: mental operations tend to persist beyond the point at which they are relevant, eg: repetition of the same words or phrases over and over again despite the interviewer's direction to stop.

- Blocking: Occurs when the thinking process stop together. It occurs in schizophrenia and anxiety states, the patients may not start with the same topic again.

\section{c) Possession and Control}

- Obsessions: persistent occurrence of ideas, thoughts, images, impulses or phobias. The patient recognizes that the ideas do not make sense and are his own (i.e. arises from inside) but occurring against his will.

- Phobias: persistent, excessive, irrational fear about a real or imaginary object, place or a situation

- Thought alienation: the patient has the experience that his thoughts are under the control of an outside agency or that others are participating in his thinking.

- Insertion: thoughts are inserted by someone

- Withdrawal: a foreign influence has withdrawn his thoughts

- Broadcasting: the patient thinks that others can read his thoughts or everyone else is thinking in union with him. Eg: in schizophrenia

\section{- Suicidal/homicidal thoughts}

\section{Rubrics related to thought}

- Mind, Thoughts, disconnected

- Thoughts, disconnected, talking while

- Thoughts, wandering, talking while 
- Thoughts, knows, thoughts of- see Clairvoyance

- Thoughts, will, thoughts separated from

- Thoughts, unpleasant subjects, haunted by

- Thoughts- persistent- murder

- Thoughts, crowding

- Thoughts, rapid

- Thoughts, ridiculous

- Thoughts, stagnation

- Thoughts, strange

- Speech, finish sentence cannot

- Speech, finish sentence cannot, jumping from thought to thought in middle of a sentence

- Delusions (658-Sub Rubrics)

- Loquacity, changing quickly from one subject to a

- Loquacity- nonsense

- Loquacity- insane

- Suicidal Disposition (91)

- Kill, Desire to be-

- Kill, Desire to -HEP, HYOS

6. Perception: is a cognitive process of becoming aware of the objects and the environment around us by way of the sense organs.

The various kinds of perceptions include visual (seeing objects), auditory (hearing sounds), tactile or haptic (feeling touch), olfactory (smelling odors), and gustatory (feeling taste).

Hallucination is a vivid sensory (perceptive) experience occurring in the absence of the corresponding external object or stimulus. In auditory hallucinations, one may hear voices or noises in the absence of the corresponding external stimulus. In visual hallucination, one may see objects in the absence of such real external objects. In olfactory hallucination, smells like burning of rubber or rags or foul gases may be experienced. Worms creeping under the skin, radiations touching the skin and the like are perceived in tactile hallucinations. In Lilliputian hallucinations, the hallucinatory objects especially the people appear much smaller. The hallucinations experiencing outside the visual field is called extra-campine hallucination. One may hear voices speaking at London when the person is in Delhi. One may have experience behind the head. In synesthetic hallucination, the real sensory experience getting in one sensory organ is experienced in another sensory modality. A music heard is experienced as beautiful colors and good taste. This is common with hallucinogens such as LSD, mescaline and cannabis. Hypnagogic hallucinations occurring as one falls to sleep and hypnopompic hallucinations occurring as one awakens from sleep are normal phenomena. In de-reality feeling the sense of the real world is disturbed. It is an -as if feeling. The environment may appear as if changed in some mysterious way (derealization) or the person may appear to him as if changed in some mysterious way (depersonalization)

Illusions are misinterpretations of perceptions (eg: shadows are mistaken for frightening figures), a rope may be misperceived as a snake. The illusion can be corrected by verifying the truth by close examination.

In micropsia the real object or figure appear smaller than what they actually are and in macropsia it is reverse. They are usually found in temporal lobe epilepsy.

Déjà $v u$ : feeling of familiarity with unfamiliar things.

Deja pense: a patient's feeling, verging on certainty that he has already thought of the matter.

Deja enterndu: the feeling that one had at some prior time heard or perceived what one is hearing in the present.

Deja raconte: a forgotten experience, particularly from the distant past, is recalled and the individual feels as if he had known all the time that the experience had been told to him.

Deja eprouve: the feeling that an act or experience in which the subject has never in fact engaged, has already been carried out by him.

Deja fait: what is happening to the patient now has happened to him before

Jamais vu: an erroneous feeling or conviction that one has never seen anything like that before (i.e. feeling of unfamiliarity)

\section{Rubrics related to perception}

- Hallucinations (Cross Reference)- See Delusions

- Delusions, sees specters, ghosts, spirits

- Delusions, voices hearing

- Delusions

- Illusions (Cross Reference)- See Delusions

- Delusions, hearing, illusions of

7. Orientation: it is awareness about time, place and person. When it is impaired, it is usually in the order of time, then place and then person and when it is regained it is in the reverse order.

- In time: It is tested by asking time of day, day of week, date and year.it may be: does the patient know his duration of hospitalization? Impaired in delirium, dementia etc.

- In place: Does patient know where he or she is? asking the address of patient's house or office

- In person: Does patient know who the examinee is, the names and relations of the familiar attendants to him

\section{Rubrics related to orientation}

Chaotic, confused behaviour

Confusion of mind, knows not where she is

8. Memory: The ability, process or act of remembering or recalling and especially the ability to reproduce what has been learned or experienced. The memory can be

Remote memory: the ability to recall information what was experienced in the distant past. It is tested by asking the childhood data, important events known to have occurred when the patient was younger or free of illness, personal matters. Take into account the patient's age and normal intelligence and which for gaps of knowledge, confabulation or perseveration

Recent past memory: The past few months

Recent memory: the past few days, recall of what was done yesterday, the day before, what was eaten for breakfast, lunch, dinner etc.

Immediate retention and recall: The ability to register information. Ability to repeat 5-6 figures after examiner dictates them-first forward and then backward, then after a few minutes interruption. The examiner may show 4-5 objects (known to patient) after asking him to remember and then recall after 4-5 minutes 


\section{Rubrics related to memory}

- Memory, active,

- Memory, confused

- Memory, destroyed absolutely (KB)

- Memory, false memory (Limest-b),

- Memory, Loss of memory (88),

- Short term memory, See- Memory, weakness-factsrecent, just happened, just read

- poor, Weakness of memory (392)

- Forgetful (333),

9. General information and intelligence (GII): This can be assessed clinically by knowing his adaptive skills, general knowledge, occupational adjustments, educational achievement, motor skills, management of finance, household and others during the interview. Intelligence can also be measured by scales of intelligence. When intelligence is not proportionately grown and developed mental retardation results. Dementia is an acquired disorder and mental retardation is a developmental disorder.

\section{Rubrics related to GII}

Intellectual

Idiocy

Imbecile

10. Attention and concentration: Conscious and willful focusing of mental energy on one object or one component of a complex experience.

- Active attention (concentration): the amount effort the patient exerts to solve a problem. It is tested by asking the patient to solve certain problems (eg: keep on subtracting 7 from 100 or months of the year backwards). It may be disturbed in mania, anxiety states, depression and those with impaired reasoning

- Passive attention: the attention which the environment draws, and the patient pays very little effort, eg: a shop on fire, or an accident. It may be disturbed in organic mental disorders

\section{Rubrics related to attention and concentration}

- Concentration, active,

- Concentration, difficult (412),

- Concentration, impossible (7)- Carc, Cadmium met

11. Abstract Thinking: It is determined by asking the meaning of common idioms, proverbs and similarities and differences between objects in the same class, eg: between ball and orange: fly and aeroplane etc. Simple questions should be asked first to reduce performance anxiety. Abstraction does not develop fully until early adolescence, is closely linked to educational accomplishment, and may appear impaired if the person is not fluent in language used. Abstraction is impaired in Schizophrenia, Mental Retardation etc.

\section{Rubrics related to abstract thinking}

Thinking, abstract thinking, images, abstract, concepts become like Thinking, Abstract thinking, inability for

12. Judgment: It is the capacity to draw direct conclusions from the material acquired by experience. It is impaired in psychoses.

Test judgement: (patient's prediction what he or she would do in imaginary situations). The type of judgement is assessed by giving a patient a particular situation and judging his response, eg: asking a patient "suppose the room in which you are sitting catches fire, what will you do?". A patient with suicidal tendencies may say "I would like to die" or a patient with delusions of grandiosity may say "the fire can do no harm to me"

Social judgement: (subtle manifestations of behavior that are harmful to the patient and contrary to acceptable behavior in the culture). This can be assessed from the history by noting his attitude towards social, financial, domestic or ethical problems. A patient with abnormal abusive violent behavior obviously has impaired judgement. Judgement should always be tested at the time of discharge from the hospital by asking him, what does he propose to do when he is discharged from the ward. A deluded patient with incomplete improvement may say, "I will take revenge from that person".

\section{Rubrics related to judgement}

Mind, Hatred, and revenge

13. Insight: It refers to subjective awareness of the pathological nature of psychiatric symptoms and behavioral disturbances. Lack of insight is characteristic of psychoses.

The level of insight must be marked from 1-6

1. Complete denial or lack of insight

2. Slight awareness of being sick and needing help but denying it at the same time

3. Awareness of being sick but attributable to external factors

4. Awareness that illness is due to something unknown in patient

5. Intellectual insight: Awareness of being ill and that the symptoms/ failure in social adjustment are due to own particular irrational feelings/thoughts, yet does not apply this knowledge to the current/failure experiences.

6. True emotional insight: Awareness leads to significant basic changes in the future behavior and personality.

- Anosognosia: the denial of illness or lack of insight seen in right parietal lobe lesions

\section{Rubrics related to insight and judgment}

- Well, says he is well, sick, when very- ARN, OP, PULS

- Delusions- well, he is

- Refusing medicine

- Refusing treatment

Table 1: Components of Mental Status Examination

\begin{tabular}{|c|c|c|c|}
\hline & Components & Observed & Inquired \\
\hline 1 & General Appearance \& Behaviour (Gab) & $\checkmark$ & \\
\hline 2 & Psychomotor Activity (Pma) & $\checkmark$ & \\
\hline 3 & Speech & $\checkmark$ & $\checkmark$ \\
\hline 4 & Affect & $\checkmark$ & $\checkmark$ \\
\hline 5 & Mood & & $\checkmark$ \\
\hline 6 & Thought & \\
\hline
\end{tabular}




\begin{tabular}{|c|c|c|c|}
\hline 7 & Perception & & $\checkmark$ \\
\hline 8 & Orientation & & $\checkmark$ \\
\hline 9 & Memory & & $\checkmark$ \\
\hline 10 & General Information \& Intelligence & & $\checkmark$ \\
\hline 11 & Attention \& Concentration & & $\checkmark$ \\
\hline 12 & Abstraction & & $\checkmark$ \\
\hline 13 & Judgement & $\checkmark$ & $\checkmark$ \\
\hline 14 & Insight & & $\checkmark$ \\
\hline
\end{tabular}

\section{Conclusion}

Understanding different components of MSE helps in eliciting different mental symptoms of the patient systematically and converting such symptoms into appropriate rubrics will help in erecting the totality and arriving at the similimum in Homoeopathic prescribing.

\section{Acknowledgements}

We convey our sincere thanks to Dr. N.D. Mohan, MD (Psy), HOD, Dept. Of Psychiatry, National Homoeopathy Research Institute in Mental Health, Kottayam for enlightening us on the subject.

\section{References}

1. Martin DC. The Mental Status Examination. In: Walker HK, Hall WD, Hurst JW, eds. Clinical Methods: The History, Physical, and Laboratory Examinations. 3rd ed. Boston: Butterworths 1990.

2. Neto HG, Estellita-Lins CE, Lessa JLM, Cavalcanti MT. Mental State Examination and Its ProceduresNarrative Review of Brazilian Descriptive Psychopathology. Front Psychiatry 2019;10:77. Published 2019 Mar 5. doi:10.3389/fpsyt.2019.00077

3. Blaabjerg ES, Hemmingsen R, Høegh E, Wang AG, Gefke M, Arnfred S. Variability between psychiatrists on domains of the mental status examination. Nordic journal of psychiatry 2020;74(4):287-292.

4. Norris D, Clark MS, Shipley S. The Mental Status Examination. Am Fam Physician 2016;94(8):635-641.

5. Guy E Brannon, MD: Chief Editor: David Bienenfeld MD: History and mental status examination cited from https://www.coursehero.com/file/76701696/Historyand-Mental-Status-Examination-Updateddocx/

6. Soltan M, Gurguis J, How to approach the mental state examination, BMJ 2017, 357 doi: https://doi.org/10.1136/sbmj.j1821 (Published 08 May 2017)

7. Snyderman D, Rovner B. Mental status exam in primary care: a review. Am Fam Physician. 2009;80(8):809-814.

8. American Psychiatric Association. Diagnostic and Statistical Manual of Mental Disorders (5 ${ }^{\text {th }}$ ed.) (DSM 5). Arlington, VA, APA 2013.

9. Sadock JB, Sadock VA, Ruiz P. Kaplan \&amp; Sadock's Comprehensive textbook of Psychiatry. Philadelphia, Lippincott Williams \& amp; Wilkins, $10^{\text {th }}$ edition 2009.

10. Kent JT, Lectures on Homoeopathic philosophy, Khrhart \&Karl 1900.

11. Hahnemann, Samuel. Organon of medicine. London: Headland 1849.

12. Schroyens F, RADAR Opus, treasure edition 2009v. 